\title{
Quasicomplex $\mathcal{N}=2, d=1$ Supersymmetric Sigma Models
}

Evgeny A. IVANOV ${ }^{\dagger}$ and Andrei V. SMILGA

$\dagger$ Bogoliubov Laboratory of Theoretical Physics, JINR, 141980 Dubna, Russia

E-mail: eivanov@theor.jinr.ru

$\ddagger$ SUBATECH, Université de Nantes, 4 rue Alfred Kastler, BP 20722, Nantes 44307, France ${ }^{1}$

E-mail: smilga@subatech.in2p3.fr

Received June 30, 2013, in final form November 13, 2013; Published online November 18, 2013

http://dx.doi.org/10.3842/SIGMA.2013.069

\begin{abstract}
We derive and discuss a new type of $\mathcal{N}=2$ supersymmetric quantum mechanical sigma models which appear when the superfield action of the $(\mathbf{1}, \mathbf{2}, \mathbf{1})$ multiplets is modified by adding an imaginary antisymmetric tensor to the target space metric, thus completing the latter to a non-symmetric Hermitian metric. These models are not equivalent to the standard de Rham sigma models, but are related to them through a certain special similarity transformation of the supercharges. On the other hand, they can be obtained by a Hamiltonian reduction from the complex supersymmetric $\mathcal{N}=2$ sigma models built on the multiplets $(\mathbf{2}, \mathbf{2}, \mathbf{0})$ and describing the Dolbeault complex on the manifolds with proper isometries. We study in detail the extremal two-dimensional case, when the target space metric is defined solely by the antisymmetric tensor, and show that the corresponding quantum systems reveal a hidden $\mathcal{N}=4$ supersymmetry.
\end{abstract}

Key words: supersymmetry; geometry; superfield

2010 Mathematics Subject Classification: 81Q60; 81T60; 14F40

\section{Introduction}

Supersymmetric quantum mechanical (SQM) sigma models have been studied for more than 30 years. The Lagrangian of the simplest and most known model of this type has the form [12]

$$
L=-\frac{1}{2} \int d \bar{\theta} d \theta g_{M N}(X) D X^{M} \bar{D} X^{N}
$$

where $X^{M}$ are the $(\mathbf{1}, \mathbf{2}, \mathbf{1})$ superfields of one-dimensional $\mathcal{N}=2$ supersymmetry $^{2}$,

$$
X^{M}=x^{M}+\theta \psi^{M}+\bar{\psi}^{M} \bar{\theta}+F^{M} \theta \bar{\theta},
$$

and $D, \bar{D}$ are the $d=1$ superspace covariant derivatives,

$$
D=\frac{\partial}{\partial \theta}-i \bar{\theta} \frac{\partial}{d t}, \quad \bar{D}=-\frac{\partial}{\partial \bar{\theta}}+i \theta \frac{\partial}{d t} .
$$

In components, this gives

$$
\begin{aligned}
L= & \frac{1}{2} g_{M N}\left[\dot{x}^{M} \dot{x}^{N}+F^{M} F^{N}+i\left(\bar{\psi}^{M} \nabla \psi^{N}-\nabla \bar{\psi}^{M} \psi^{N}\right)\right] \\
& +\Gamma_{M, P Q} F^{M} \psi^{P} \bar{\psi}^{Q}+\frac{1}{2}\left(\partial_{M} \partial_{Q} g_{P N}\right) \bar{\psi}^{M} \psi^{N} \bar{\psi}^{P} \psi^{Q} .
\end{aligned}
$$

\footnotetext{
${ }^{1}$ On leave of absence from ITEP, Moscow, Russia.

${ }^{2}$ We use the notation of [19] where the numerals count the numbers of the dynamic bosonic, dynamic fermionic, and auxiliary bosonic fields.
} 
Here $\Gamma_{M, P Q}$ are the Christoffel symbols and $\nabla \psi^{N}=\dot{\psi}^{N}+\Gamma_{P Q}^{N} \dot{x}^{P} \psi^{Q}$. After eliminating the auxiliary fields $F^{M}$, the Lagrangian acquires the form

$$
L=\frac{1}{2} g_{M N}\left[\dot{x}^{M} \dot{x}^{N}+i\left(\bar{\psi}^{M} \nabla \psi^{N}-\nabla \bar{\psi}^{M} \psi^{N}\right)\right]+\frac{1}{2} R_{M N P Q} \bar{\psi}^{M} \psi^{N} \bar{\psi}^{P} \psi^{Q},
$$

where $R_{M N P Q}$ is the Riemann tensor.

The geometrical interpretation of this model is also well known. Its Hilbert space can be mapped onto the space of differential forms such that supercharges can be interpreted as the exterior derivative operator and its complex conjugate. Thereby, the system amounts to the de Rham complex [23, 24].

Another complex known to mathematicians, the Dolbeault complex which is defined on a complex manifold and involves only holomorphic $(p, 0)$-forms, can also be formulated as a supersymmetric sigma model. Its superfield action $[17,18]$ involves $(\mathbf{2 , 2 , 0})$ superfields,

$$
Z^{j}=z^{j}+\sqrt{2} \theta \psi^{j}-i \theta \bar{\theta} \dot{z}^{j}, \quad \bar{Z}^{\bar{j}}=\bar{z}^{\bar{j}}-\sqrt{2} \bar{\theta} \bar{\psi}^{\bar{j}}+i \theta \bar{\theta} \dot{\bar{z}} \bar{j},
$$

$j, \bar{j}=1, \ldots, n, \bar{D} Z=D \bar{Z}=0$.

In the simplest case,

$$
S=-\frac{1}{4} \int d t d^{2} \theta h_{j \bar{k}}(Z, \bar{Z}) D Z^{j} \bar{D} \bar{Z}^{\bar{k}}
$$

where $h_{j \bar{k}}$ is a Hermitian complex metric.

Coming back to (1.1), it describes the de Rham complex provided the tensor $g_{M N}$ is symmetric and has the meaning of the target space metric. However, nothing prevents us from asserting that this tensor has also an imaginary antisymmetric part (we should keep $g_{M N}$ Hermitian, otherwise we would lose the reality of the Lagrangian and Hermiticity of the Hamiltonian). For some reasons, the Lagrangians of this type and the associated quantum-mechanical systems never got any detailed attention in the literature ${ }^{3}$. Our study is intended to fill up this gap.

There are relationships of our model and the SQM models previously considered. In particular, we demonstrate that our model can be recovered by an appropriate Hamiltonian reduction from the general complex $\mathcal{N}=2 \mathrm{SQM}$ model possessing some shift isometries and dealing with the manifolds of the doubled real dimension. So, from the geometric point of view, the considered class of $\mathcal{N}=2 \mathrm{SQM}$ models can be interpreted as a restriction of the Dolbeault complex to the real slice of the original complex target space.

Second, though the complex thus obtained does not coincide with the de Rham complex describing the standard $(\mathbf{1}, \mathbf{2}, \mathbf{1})$ SQM models with symmetric target space metric, it can be obtained from the latter by a similarity transformation of the holomorphic supercharges. We demonstrate it explicitly for the simplest 2-dimensional model of this type with the "primordial" metric $g_{M N}=\delta_{M N}+i \epsilon_{M N} b(x)$. The supercharges that we derive are related by a similarity transformation to the de Rham supercharges with a conformally flat "associated" metric

$$
h_{M N}=\sqrt{1-b^{2}} \delta_{M N}
$$

For this simple model, we derive the explicit expressions for the quantum supercharges and the Hamiltonian. The latter does not coincide with the standard de Rham Hamiltonian (i.e. the

\footnotetext{
${ }^{3}$ The existence of such models was mentioned in $[4,13,17]$. In particular, it was noticed in [13] that they cannot be obtained by dimensional reduction from the Lorentz-covariant $\mathcal{N}=1, d=2$ sigma models, as distinct from those based on the Lagrangians (1.1) with symmetric metric (the $\mathcal{N}=2, d=1$ multiplets (1, 2, 1) and $(\mathbf{2}, \mathbf{2}, \mathbf{0})$ were termed in [13] as $N=2 a$ and $N=2 b$, respectively). The precise relation with the general $\mathcal{N}=1$, $d=2$ sigma model Lagrangian is given in Appendix A.
} 
covariant Laplacian acting on the forms). This stems from the fact that the similarity transformations for $Q$ and $\bar{Q}$ that we use are different (see equation (3.13) below). We demonstrate that the system exhibits hidden $\mathcal{N}=4$ supersymmetry at the quantum level: the relevant energy levels reveal 4-fold degeneracy. We dwell on a special choice of the function $b(x)$ describing the dynamics on the 2-sphere $S^{2}$. The detailed analysis of this new $\mathcal{N}=2$ (and $\mathcal{N}=4$ ) SQM model is performed in the Appendix B. In a certain limit, the Hamiltonian thus obtained is related by a similarity transformation to the square of the Dirac operator on $S^{2}$.

\section{The general model}

Our starting point is the superfield Lagrangian (1.1) where the tensor $g_{M N}$ involves now an antisymmetric part,

$$
g_{M N}=g_{(M N)}+i b_{[M N]},
$$

with real $g_{(M N)}, b_{[M N]}$. Plugging there the superfield (1.2), we derive the component Lagrangian

$$
\begin{aligned}
L= & \frac{1}{2} g_{(M N)}\left(\dot{x}^{M} \dot{x}^{N}+F^{M} F^{N}\right)+b_{[M N]} \dot{x}^{M} F^{N}+\frac{i}{2} g_{(M N)}\left(\bar{\psi}^{N} \nabla \psi^{M}-\nabla \bar{\psi}^{N} \psi^{M}\right) \\
& -\frac{1}{2} b_{[M N]}\left(\bar{\psi}^{N} \dot{\psi}^{M}-{ }^{-} \dot{\psi} \psi \psi^{M}\right)-\frac{1}{2} \partial_{P} \partial_{Q}\left(g_{(M N)}+i b_{[M N]}\right) \psi^{M} \bar{\psi}^{N} \psi^{P} \bar{\psi}^{Q} \\
& +G_{M, P Q} F^{M} \psi^{P} \bar{\psi}^{Q}-\frac{1}{2}\left(\partial_{M} b_{[N P]}+\partial_{N} b_{[M P]}\right) \dot{x}^{P} \psi^{M} \bar{\psi}^{N},
\end{aligned}
$$

where

$$
\begin{aligned}
G_{M, P Q} & =\Gamma_{M, P Q}-\frac{i}{2}\left(\partial_{M} b_{[P Q]}+\partial_{P} b_{[Q M]}+\partial_{Q} b_{[M P]}\right) \\
& =\frac{1}{2}\left(\partial_{P} g_{M Q}+\partial_{Q} g_{P M}-\partial_{M} g_{P Q}\right)
\end{aligned}
$$

are complex Christoffel symbols calculated for the complex metric (2.1), while $\Gamma_{M, P Q}$ are the standard Christoffels for $g_{(M N)}$

$$
\Gamma_{M, P Q}=\frac{1}{2}\left[\partial_{P} g_{(M Q)}+\partial_{Q} g_{(M P)}-\partial_{M} g_{(P Q)}\right], \quad \nabla \psi^{M}=\dot{\psi}^{M}+\Gamma_{N Q}^{M} \dot{x}^{N} \psi^{Q} .
$$

The Lagrangian (2.2) is invariant (modulo a total derivative) under the following $\mathcal{N}=2$ supersymmetry transformations

$$
\begin{array}{ll}
\delta x^{M}=-\epsilon \psi^{M}+\bar{\epsilon} \bar{\psi}^{M}, & \delta \psi^{M}=\bar{\epsilon}\left(i \dot{x}^{M}-F^{M}\right), \\
\delta \bar{\psi}^{M}=-\epsilon\left(i \dot{x}^{M}+F^{M}\right), & \delta F^{M}=i\left(\epsilon \dot{\psi}^{M}+\bar{\epsilon} \dot{\bar{\psi}}^{M}\right) .
\end{array}
$$

It is worth noting that the whole Lagrangian (2.2) can be written through the Hermitian metric $g_{M N}$ because it is the latter that enters the generalized superfield Lagrangian (1.1) we started with. However, the terms with the derivatives of $b_{[M N]}$ cannot be fully accommodated through the generalized Christoffel symbols (2.3). For instance, the full part bilinear in the fermionic fields can be written as

$$
\frac{i}{2} g_{M N}\left(\bar{\psi}^{N} \dot{\psi}^{M}-\dot{\bar{\psi}}^{N} \psi^{M}\right)-\frac{i}{2}\left(\partial_{S} g_{P N}-\partial_{N} g_{S P}\right) \dot{x}^{P} \psi^{S} \bar{\psi}^{N}
$$

and the second piece cannot be written through $G_{M, P Q}$. This means that the 2-form field $b_{[M N]}$ cannot be interpreted as a potential of some closed torsion. Respectively, the Lagrangian (2.2) and the original superfield Lagrangian do not reveal any extra target space gauge symmetry 
associated with $b_{[M N]}$. The only target space gauge symmetry of (1.1) with the Hermitian metric (2.1) is the diffeomorphisms

$$
\delta X^{M}=\Lambda^{M}(X), \quad \delta g_{M N}=-\partial_{M} \Lambda^{P} g_{P N}-\partial_{N} \Lambda^{P} g_{M P},
$$

like in the case of symmetric metric. It is curious that the Hermitian non-symmetric metric, with the antisymmetric part having no interpretation as a gauge field, was considered by Einstein and Strauss more than 60 years ago $[7,8]$ as the basic ingredient of some generalization of the standard Einstein gravity (for a recent renewal of interest in such theories, see [2,3] and references therein).

As one more comment, we mention the extreme possibility of the choice $g_{(M N)}=0$ in (2.1). In this case, the superfield Lagrangian is reduced to $\sim b_{[M N]}(X) D X^{M} \bar{D} X^{N}$, and the component Lagrangian (2.2) with $g_{(M N)}=0$ provides a new $\mathcal{N}=2$ supersymmetric extension of the Chern-Simons quantum mechanics $[6,9,11,16]$, based on the multiplet $(\mathbf{1}, \mathbf{2}, \mathbf{1})$ (in [16] only the version built on the chiral $(\mathbf{2}, \mathbf{2}, \mathbf{0})$ multiplet was considered). In such a model, with nondegenerate $b_{[M N]}$, the field $F^{M}$ plays the dynamical role of the canonical momenta for $X^{M}$ and cannot be eliminated algebraically. Though being worthy of detailed study, this option is beyond the scope of our consideration in the present paper. In what follows, we assume that $g_{(M N)} \neq 0$.

After elimination of the auxiliary field $F^{M}$ by its equation of motion

$$
F^{M}=b_{P}^{M} \dot{x}^{P}-G_{P Q}^{M} \psi^{P} \bar{\psi}^{Q}
$$

(with the indices being raised with $g^{(M P)}$ ), the Lagrangian acquires the form

$$
\begin{aligned}
L= & \frac{1}{2} g_{(M N)} \dot{x}^{M} \dot{x}^{N}-\frac{1}{2} g^{(M N)}\left(b_{[M P]} \dot{x}^{P}-G_{M, P Q} \psi^{P} \bar{\psi}^{Q}\right)\left(b_{[N S]} \dot{x}^{S}-G_{N, S T} \psi^{S} \bar{\psi}^{T}\right) \\
& +\frac{i}{2} g_{(M N)}\left(\bar{\psi}^{N} \nabla \psi^{M}-\nabla \bar{\psi}^{N} \psi^{M}\right)-\frac{1}{2} b_{[M N]}\left(\bar{\psi}^{N} \dot{\psi}^{M}-{ }^{-}{ }^{N} \psi^{M}\right) \\
& -\frac{1}{2} \partial_{P} \partial_{Q}\left(g_{(M N)}+i b_{[M N]}\right) \psi^{M} \bar{\psi}^{N} \psi^{P} \bar{\psi}^{Q}-\frac{1}{2}\left(\partial_{M} b_{[N P]}+\partial_{N} b_{[M P]}\right) \dot{x}^{P} \psi^{M} \bar{\psi}^{N} .
\end{aligned}
$$

Note that the bosonic core of the Lagrangian (2.6) is

$$
L^{\mathrm{bos}}=\frac{1}{2} G_{(M N)} \dot{x}^{M} \dot{x}^{N}, \quad G_{(M N)}=g_{(M N)}+b_{[M P]} g^{(P S)} b_{[S N]},
$$

i.e. the antisymmetric field $b_{[M P]}$ makes a non-trivial contribution to the target space metric ${ }^{4}$.

The Lagrangian (2.6) is invariant, up to a total derivative, under the nonlinear supersymmetry transformations

$$
\begin{aligned}
& \delta x^{M}=-\epsilon \psi^{M}+\bar{\epsilon} \bar{\psi}^{M}, \quad \delta \psi^{M}=\bar{\epsilon}\left(i \dot{x}^{M}-b_{P}^{M} \dot{x}^{P}+G_{P Q}^{M} \psi^{P} \bar{\psi}^{Q}\right), \\
& \delta \bar{\psi}^{M}=-\epsilon\left(i \dot{x}^{M}+b_{P}^{M} \dot{x}^{P}-G_{P Q}^{M} \psi^{P} \bar{\psi}^{Q}\right) .
\end{aligned}
$$

Using these transformations, one can derive the conserved Nöther supercharges

$$
\begin{aligned}
& Q=\psi^{M}\left[\Pi_{M}-\frac{i}{2} \partial_{M}\left(g_{(N P)}+i b_{[N P]}\right) \psi^{N} \bar{\psi}^{P}\right], \\
& \bar{Q}=\bar{\psi}^{M}\left[\Pi_{M}+\frac{i}{2} \partial_{M}\left(g_{(N P)}-i b_{[N P]}\right) \psi^{P} \bar{\psi}^{N}\right],
\end{aligned}
$$

\footnotetext{
${ }^{4}$ This metric resembles a block of the generalized bosonic metric in the so called "double field theory" (see, e.g., [15]) and the metrics obtained via $T$-duality [14]. However, it differs from such metrics by the sign before the second term. This important difference is related to the fact that our original non-symmetric metric $g_{M N}$ is Hermitian, while its counterparts in the references just mentioned are real, with the antisymmetric parts being gauge fields.
} 
where

$$
\begin{aligned}
\Pi_{M}= & g_{(M N)} \dot{x}^{N}-g^{(P N)} b_{[P M]}\left(b_{[N S]} \dot{x}^{S}-G_{N, S T} \psi^{S} \bar{\psi}^{T}\right) \\
& -\frac{i}{2}\left(\partial_{P} g_{(Q M)}-\partial_{Q} g_{(P M)}\right) \psi^{P} \bar{\psi}^{Q}-\frac{1}{2}\left(\partial_{P} b_{[Q M]}+\partial_{Q} b_{[P M]}\right) \psi^{P} \bar{\psi}^{Q}
\end{aligned}
$$

is the canonical momentum of $x^{M}, \Pi_{M}=\frac{\partial L}{\partial \dot{x}^{M}}$. It is worth noting that it is easier to derive the supercharges starting just from the off-shell Lagrangian (2.2) and transformations (2.4). The final expressions for $Q, \bar{Q}$ do not involve the auxiliary field $F^{M}$ and have the same form (2.8).

When $b_{[M N]}$ is absent, the supercharges (2.8) acquire the form

$$
Q=\psi^{M}\left[\Pi_{M}+\frac{i}{2} \Gamma_{M, N P} \psi^{N} \bar{\psi}^{P}\right], \quad \bar{Q}=\bar{\psi}^{M}\left[\Pi_{M}-\frac{i}{2} \Gamma_{M, N P} \psi^{P} \bar{\psi}^{N}\right] .
$$

These are the supercharges of the standard de Rham $\mathcal{N}=2$ sigma model written in a somewhat unusual form ${ }^{5}$. The usual form is

$$
Q=\psi^{M}\left[P_{M}-i \Omega_{M, A B} \psi_{A} \bar{\psi}_{B}\right], \quad \bar{Q}=\bar{\psi}^{M}\left[P_{M}-i \Omega_{M, A B} \bar{\psi}_{A} \psi_{B}\right],
$$

where $A, B$ are the tangent space indices, $\psi_{A}=e_{A M} \psi^{M}, g_{M N}=e_{A M} e_{A N}$, and

$$
\Omega_{M, A B}=e_{A N}\left(\partial_{M} e_{B}^{N}+\Gamma_{M T}^{N} e_{B}^{T}\right)
$$

are spin connections.

Note that the canonical momenta $P_{M}$ entering (2.10) are obtained by the variation of the Lagrangian over $\dot{x}_{M}$ with fixed $\psi_{A}, \bar{\psi}_{A}$, while $\Pi_{M}$ appearing in (2.8), (2.9) is obtained by the variation with fixed $\psi^{M}, \bar{\psi}^{M}$. These two canonical momenta are related as [10],

$$
P_{M}=\Pi_{M}-\frac{\partial \dot{\psi}_{A}}{\partial \dot{x}^{M}} \frac{\partial L}{\partial \dot{\psi}_{A}}-\frac{\partial \dot{\bar{\psi}}_{A}}{\partial \dot{x}^{M}} \frac{\partial L}{\partial \dot{\bar{\psi}}_{A}}=\Pi_{M}+\frac{i}{2}\left[\partial_{M} e_{A P} e_{A Q}-\partial_{M} e_{A Q} e_{A P}\right] \psi^{P} \bar{\psi}^{Q}
$$

For the standard sigma model, the form (2.10) is more convenient because it allows one to perform the quantization rather straightforwardly. Indeed, the "flat" fermion variables $\psi_{A}, \bar{\psi}_{A}$ constitute, together with $x^{M}, P_{M}$, the canonically conjugated pairs,

$$
\begin{aligned}
& \left\{\psi_{A}, \bar{\psi}_{B}\right\}_{\text {P.B. }}=-i \delta_{A B}, \quad\left\{x^{M}, P_{N}\right\}_{\text {P.B. }}=\delta_{N}^{M}, \\
& \left\{\psi_{A}, x^{M}\right\}_{\text {P.B. }}=\left\{\bar{\psi}_{A}, x^{M}\right\}_{\text {P.B. }}=\left\{\psi_{A}, P_{M}\right\}_{\text {P.B. }}=\left\{\bar{\psi}_{A}, P_{M}\right\}_{\text {P.B. }}=0 .
\end{aligned}
$$

When quantizing, we have to replace $P_{M} \rightarrow-i \partial / \partial_{M}, \bar{\psi}_{A} \rightarrow \partial / \partial \psi_{A}$ and to choose a particular way of ordering the momenta and coordinates. Especially convenient covariant nilpotent quantum supercharges have the same functional form as $(2.10)^{6}$. They are Hermitian-conjugated to each other, bearing in mind the factor $\sqrt{g}$ in the measure,

$$
\bar{Q}=(\operatorname{det} g)^{-1 / 2} Q^{\dagger}(\operatorname{det} g)^{1 / 2}
$$

where $Q^{\dagger}$ is related to $Q$ by a "naive" conjugation. Other orderings correspond to some extra conjugations, $Q \rightarrow e^{W} Q e^{-W}, \bar{Q} \rightarrow e^{-W} \bar{Q} e^{W}$. This is interpreted as introducing potentials on the manifold [23, 24].

\footnotetext{
${ }^{5}$ Surprisingly, we were not able to find such a simple representation for the classical supercharges of the $(\mathbf{1}, \mathbf{2}, \mathbf{1})$ $\mathcal{N}=2$ models in literature.

${ }^{6}$ See $[20]$ for general recipes of resolving the ordering ambiguities in SQM models.
} 
The variables $\psi^{M}, \bar{\psi}^{M}$ satisfy more complicated relations,

$$
\begin{aligned}
& \left\{\psi^{M}, \bar{\psi}^{N}\right\}_{\text {P.B. }}=-i g^{M N}, \\
& \left\{\Pi_{M}, \psi^{N}\right\}_{\text {P.B. }}=-\frac{1}{2} \partial_{M} g^{N Q} \psi_{Q}, \quad\left\{\Pi_{M}, \bar{\psi}^{N}\right\}_{\text {P.B. }}=-\frac{1}{2} \partial_{M} g^{N Q} \bar{\psi}_{Q} .
\end{aligned}
$$

In this case, the quantization procedure is somewhat trickier, with quantum commutators corresponding not to the Poisson brackets, but rather to the Dirac brackets.

In our case with non-vanishing $b_{[M N]}$, we can also introduce canonically conjugated flat fermion variables related to $\psi^{M}, \bar{\psi}^{M}$ by complex vielbeins, and then quantize. We will do this in the next section for the simplest nontrivial 2-dimensional model.

However, to derive the result that the model (1.1) with generic Hermitian metric $g_{M N}$ can be obtained by a Hamiltonian reduction of a certain Dolbeault sigma model (the subject of Section 4), we do not need to come to grips with quantization. The whole reasoning will be carried out at the classical level and in this case the supercharges (2.8) prove to be more convenient than the supercharges (2.10).

\section{Two-dimensional model. Similarity transformation}

The simplest nontrivial case corresponds to just two bosonic coordinates, $x^{M=1,2}$, with the flat symmetric part of the metric, $g_{(M N)}=\delta_{M N}$. In this case, all expressions are greatly simplified. In particular, one can write $b_{[M N]}=b \epsilon_{M N}$. The Christoffels (2.3) vanish. The quartic fermionic terms in the Lagrangian also vanish. The latter acquires the form

$$
\begin{aligned}
L= & \frac{1}{2}\left(1-b^{2}\right) \dot{x}^{M} \dot{x}^{M}-\frac{i}{2}\left(\delta_{M N}+i b \epsilon_{M N}\right)\left(\dot{\psi}^{M} \bar{\psi}^{N}-\psi^{M} \dot{\bar{\psi}}^{N}\right) \\
& -\frac{1}{2}\left[\partial_{M} b \epsilon_{N P}+\partial_{N} b \epsilon_{M P}\right] \dot{x}^{P} \psi^{M} \bar{\psi}^{N} .
\end{aligned}
$$

We will assume $b^{2}<1$. The degenerate case $b^{2}=1$ when the kinetic term vanishes is discussed at the end of this section. The Lagrangian is then reduced to (3.17). When $b^{2}>1$, the kinetic term has a ghost signature, which requires a special analysis.

The relevant classical supercharges read

$$
Q=\psi^{M}\left[\Pi_{M}+\frac{1}{2} \partial_{M} b \epsilon_{N P} \psi^{N} \bar{\psi}^{P}\right], \quad \bar{Q}=\bar{\psi}^{M}\left[\Pi_{M}-\frac{1}{2} \partial_{M} b \epsilon_{N P} \psi^{N} \bar{\psi}^{P}\right] .
$$

In this case, the complex vielbeins transforming the variables $\psi^{M}, \bar{\psi}^{M}$ into canonically conjugated pairs can be found explicitly,

$$
\begin{aligned}
& \psi^{M}=e_{A}^{M} \psi_{A}, \quad \bar{\psi}^{M}=\bar{e}_{A}^{M} \bar{\psi}_{A}, \quad \psi_{A}=e_{A M} \psi^{M}, \quad \bar{\psi}_{A}=\bar{e}_{A M} \bar{\psi}^{M}, \\
& e_{A M}=g_{+} \delta_{A M}-i g_{-} \epsilon_{A M}, \quad \bar{e}_{A M}=g_{+} \delta_{A M}+i g_{-} \epsilon_{A M}, \\
& e_{A}^{M}=e_{A M} / \sqrt{1-b^{2}}, \quad \bar{e}_{A}^{M}=\bar{e}_{A M} / \sqrt{1-b^{2}}
\end{aligned}
$$

where

$$
g_{ \pm}=\frac{1}{2}(\sqrt{1+b} \pm \sqrt{1-b}) .
$$

These vielbeins satisfy the relations

$$
e_{A M} \bar{e}_{A N}=\delta_{M N}+i b \epsilon_{M N}, \quad e_{A M} e_{B}^{M}=\delta_{A B}, \quad e_{A M} e_{A}^{N}=\delta_{M}^{N}, \quad \text { and } \quad \text { c.c. }
$$


The Lagrangian (3.1) can then be rewritten as

$$
L=\frac{1}{2}\left(1-b^{2}\right) \dot{x}^{M} \dot{x}^{M}-\frac{i}{2}\left(\dot{\psi}_{A} \bar{\psi}_{A}-\psi_{A} \dot{\bar{\psi}}_{A}\right)-\frac{1}{2}\left[\partial_{M} b \epsilon_{N P}+\partial_{N} b \epsilon_{M P}\right] \dot{x}^{P} e_{A}^{M} \bar{e}_{B}^{N} \psi_{A} \bar{\psi}_{B}
$$

(note that the vielbein time derivatives coming from the two parts of the fermion kinetic term are canceled amongst each other). With this form of the Lagrangian, it is obvious that $\psi_{A}, \bar{\psi}_{B}$ and $P_{M}, x^{N}$ indeed constitute the mutually commuting canonical pairs.

The classical supercharges (3.2) can be expressed via the fermion variables with the tangent space indices. The direct substitution gives

$$
\begin{aligned}
& Q=\psi_{A} e_{A}^{M}\left[\Pi_{M}+\frac{\partial_{M} b}{2\left(1-b^{2}\right)}\left(\epsilon_{B C}+i b \delta_{B C}\right) \psi_{B} \bar{\psi}_{C}\right], \\
& \bar{Q}=\bar{\psi}_{A} \bar{e}_{A}^{M}\left[\Pi_{M}-\frac{\partial_{M} b}{2\left(1-b^{2}\right)}\left(\epsilon_{B C}+i b \delta_{B C}\right) \psi_{B} \bar{\psi}_{C}\right] .
\end{aligned}
$$

An important point is that, in the considered case, due to the specific structure of the vielbeins, the following equality holds

$$
P_{M}=\Pi_{M}+\frac{i}{2}\left[\left(\partial_{M} e_{P}^{A}\right) \bar{e}_{Q}^{A}-\left(\partial_{M} \bar{e}_{Q}^{A}\right) e_{P}^{A}\right] \psi^{P} \bar{\psi}^{Q}=\Pi_{M} .
$$

It is a straightforward exercise to make sure that these supercharges form the standard $\mathcal{N}=2$, $d=1$ superalgebra

$$
\{Q, Q\}_{\text {P.B. }}=\{\bar{Q}, \bar{Q}\}_{\text {P.B. }}=0, \quad\{Q, \bar{Q}\}_{\text {P.B. }}=-2 i H^{\mathrm{cl}},
$$

where $H^{\mathrm{cl}}$ is the classical Hamiltonian corresponding to the Lagrangian (3.1) (or (3.5)),

$$
H^{\mathrm{cl}}=\frac{1}{2\left(1-b^{2}\right)}\left[\Pi_{M}+\frac{1}{2}\left(\partial_{T} b \epsilon_{N M}+\partial_{N} b \epsilon_{T M}\right) \psi^{T} \bar{\psi}^{N}\right]^{2} .
$$

We notice now that, using antisymmetry of the expressions like $\psi_{A} \psi_{B}$ under the permutation $A \leftrightarrow B$ and 2-dimensional specifics, one can get rid of the structure $\delta_{B C}$ in the supercharges (3.6) and rewrite them as

$$
Q=\psi_{A} e_{A}^{M}\left(\Pi_{M}-i \Omega_{M, B C} \psi_{B} \bar{\psi}_{C}\right), \quad \bar{Q}=\bar{\psi}_{A} \bar{e}_{A}^{M}\left(\Pi_{M}-i \bar{\Omega}_{M, B C} \bar{\psi}_{B} \psi_{C}\right)
$$

with

$$
\Omega_{M, B C}=\frac{\epsilon_{B C}}{2\left(1-b^{2}\right)}\left(b \epsilon_{N M} \partial_{N} b+i \partial_{M} b\right) .
$$

This object has an interesting geometric interpretation. It is just the spin connection for the complex vielbeins $e_{A M}, e_{B}^{N}$ defined in (3.4). It can be computed by the standard formulae

$$
\begin{aligned}
\Omega_{M, A B} & =e_{A N}\left(\partial_{M} e_{B}^{N}+\hat{\Gamma}_{M T}^{N} e_{B}^{T}\right)=e_{C M} \Omega_{C, A B}, \\
\Omega_{C, A B} & =e_{B}^{M} e_{A}^{N} \partial_{[M} e_{C N]}+e_{B}^{M} e_{C}^{N} \partial_{[M} e_{A N]}+e_{C}^{M} e_{A}^{N} \partial_{[M} e_{B N]} \\
& =\frac{1}{2\left(1-b^{2}\right)} \epsilon_{A B}\left(i e_{C}^{M} \partial_{M} b+b e_{D}^{M} \partial_{M} b \epsilon_{D C}\right) .
\end{aligned}
$$

Here, $\hat{\Gamma}_{M T}^{N}$ is the standard Levi-Civita connection for the real conformally flat metric $h_{M N}=$ $e_{A M} e_{A N}=\sqrt{1-b^{2}} \delta_{M N}$ as in (1.3). 
Consider the real part of the spin connection (3.9). One observes that it can be interpreted as the standard spin connection (2.11) for the same conformally flat metric $h_{M N}$, but with the naturally chosen real vielbeins

$$
\tilde{e}_{A M}=\left(1-b^{2}\right)^{1 / 4} \delta_{A M} .
$$

Being truncated in this way, the supercharges (3.8) coincide with the supercharges of the standard $(\mathbf{1}, \mathbf{2}, \mathbf{1})$ supersymmetric sigma model $(2.10)$, though involving the new metric $h_{M N}$, equation (1.3), which is different from (but conformal to) the bosonic target metric in (3.1). So the quantum version of these supercharges describes the de Rham complex associated with the metric $h_{M N}$.

Now, come back to the full expressions (3.8). They are classical. Once again, to obtain the quantum supercharges, one should order the fermion operators in a certain particular way. Let us do it in the same way as for the standard de Rham supercharges. We thus write

$$
Q^{\mathrm{qu}}=-i \psi_{A}\left(e_{A}^{M} \partial_{M}+\Omega_{A, B C} \bar{\psi}_{B} \psi_{C}\right), \quad \bar{Q}^{\mathrm{qu}}=-i \bar{\psi}_{A}\left(\bar{e}_{A}^{M} \partial_{M}+\bar{\Omega}_{A, B C} \psi_{B} \bar{\psi}_{C}\right) .
$$

One easily checks that these supercharges are nilpotent and hence satisfy the standard quantum $\mathcal{N}=2$ superalgebra. The anticommutator $\left\{\bar{Q}^{\text {qu }}, Q^{\text {qu }}\right\}$ gives the quantum Hamiltonian $H^{\text {qu }}$ :

$$
\begin{aligned}
H^{\mathrm{qu}}= & \frac{1}{2\left(1-b^{2}\right)}\left[-\partial_{M} \partial_{M}+\frac{1}{i}\left(\partial_{T} b \epsilon_{N M}+\partial_{N} b \epsilon_{T M}\right) e_{A}^{T} \bar{e}_{B}^{N} \psi_{A} \bar{\psi}_{B} \partial_{M}\right. \\
& \left.+\frac{1}{2}\left(\partial_{T} b \partial_{M} b\right) e_{A}^{T} \bar{e}_{B}^{M} e_{D}^{S} \bar{e}_{C}^{S} \psi_{A} \psi_{D} \bar{\psi}_{B} \bar{\psi}_{C}\right] \\
& -\frac{1}{2}\left(\bar{e}_{A}^{M} \partial_{M} e_{A}^{N}+e_{A}^{N} \bar{\Omega}_{C, C A}\right) \partial_{N}+\frac{1}{2}\left(e_{A}^{M} \partial_{M} \bar{\Omega}_{C, B C}-\bar{e}_{D}^{M} \partial_{M} \Omega_{B, A D}\right) \psi_{A} \bar{\psi}_{B}
\end{aligned}
$$

An important observation is that the supercharges (3.11) can be obtained from the quantum supercharges of the de Rham complex with the metric (1.3) and vielbeins (3.10) by a similarity transformation

$$
Q^{\mathrm{qu}}=R^{-1} Q^{\mathrm{de} \text { Rham }} R, \quad \bar{Q}^{\mathrm{qu}}=R \bar{Q}^{\text {de Rham }} R^{-1},
$$

with

$$
R=\exp \left\{\frac{i}{4} \ln \frac{1+b}{1-b} \epsilon_{A B} \psi_{A} \bar{\psi}_{B}\right\} .
$$

The operator (3.14) is not unitary, $R^{\dagger}=R$ rather than $R^{-1}$, and hence $Q^{\text {qu }}$ and $\bar{Q}^{\text {qu }}$ are rotated in a different manner. The relations (3.13), (3.14) imply that the property (2.13) that holds for the de Rham supercharges holds as well for $Q^{\text {qu }}, \bar{Q}^{\text {qu }}$. In other words, the supercharges (3.13) are mutually Hermitian with the measure $\sqrt{h}=\sqrt{1-b^{2}}$.

By construction, the Hamiltonian (3.12) is Hermitian with the same measure. It does not seem to be related, however, to the de Rham Hamiltonian (the covariant Laplacian) by any similarity transformation and has, as we will see, a distinct spectrum. In the sectors $F=0,2$, the Hamiltonian is reduced to the simple expressions

$$
\begin{aligned}
& 2 H^{F=0}=\frac{1}{1-b^{2}} P_{M}^{2}-\frac{i b \partial_{M} b+\epsilon_{P M} \partial_{P} b}{\left(1-b^{2}\right)^{2}} P_{M}, \\
& 2 H^{F=2}=\frac{1}{1-b^{2}} P_{M}^{2}-\frac{i b \partial_{M} b-\epsilon_{P M} \partial_{P} b}{\left(1-b^{2}\right)^{2}} P_{M} .
\end{aligned}
$$

It is clear that for any eigenfunction $\Psi^{F=0}$ of $H^{F=0}$, the complex conjugate function $\Psi^{F=2}=$ $\left(\Psi^{F=0}\right)^{\star}$ is an eigenfunction of $H^{F=2}$ with the same eigenvalue. Supersymmetry dictates that 
the states $Q^{\mathrm{qu}} \Psi^{F=0}$ and $\bar{Q}^{\text {qu }} \Psi^{F=2}$ have also the same energy. Thus, in this case, the energy levels display a 4 -fold degeneracy and, hence, the system enjoys in fact an extended $\mathcal{N}=4$ supersymmetry. One can remind here that any 2-dimensional manifold is Kähler and hence the de Rham complex can be extended to the Kähler-de Rham complex with extended supersymmetry. There is no reason to believe, however, that such an extended supersymmetry is also valid in the generic higher-dimensional case.

Note that the $\mathcal{N}=2$ superfield action corresponding to the Lagrangian (1.1) with $g_{M N}=$ $\delta_{M N}+i b(X) \epsilon_{M N}, M, N=1,2$, reveals no any obvious second $\mathcal{N}=2$ supersymmetry which would complete the manifest $\mathcal{N}=2$ one to $\mathcal{N}=4$. So the $\mathcal{N}=4$ supersymmetry we have observed either is realized by highly nonlinear superfield transformations involving the target potential $b(X)$, or is a pure quantum-mechanical phenomenon, like in [21]. In any case, the underlying $\mathcal{N}=4$ supermultiplet is an on-shell version of the multiplet $(\mathbf{2}, \mathbf{4}, \mathbf{2})$, and its field content is the same as for two $\mathcal{N}=2$ multiplets $(\mathbf{1}, \mathbf{2}, \mathbf{1})^{7}$.

A non-triviality of the quantum problem considered in this section is the existence of four different metrics which should not be confused with each other. For reader's convenience, we thus reiterate.

- First, there is the primordial complex Hermitian metric (2.1). Its geometric meaning will be clarified in the next section.

- The symmetric part $g_{(M N)}$ of this complex metric does not have a special meaning (at least, we do not see it), but it enters the formulae (2.6), (2.7) in Section 2.

- Next, there is the "kinetic metric" (2.7) that determines the bosonic dynamics. It is constructed from both $g_{(M N)}$ and the antisymmetric potential $b_{[M N]}$. In the two-dimensional case, it boils down to $h_{M N}^{\text {kinetic }}=\left(1-b^{2}\right) \delta_{M N}$.

- Finally, there is an "associated metric" - the metric of the de Rham complex with supercharges related to our supercharges by a similarity transformation. In the 2-dimensional case, we have derived $h_{M N}^{\text {associated }}=\sqrt{1-b^{2}} \delta_{M N}$.

A detailed analysis of the spectrum of the Hamiltonian (3.12) in the simple case when the kinetic metric $h_{M N} \propto \delta_{M N} /\left(1+x_{P}^{2}\right)^{2}$ describes the 2-sphere $S^{2}$ is performed in the Appendix B.

As was mentioned above, the whole consideration above was performed assuming $b^{2}<1$. When $b^{2}>1$, the spectrum of the Hamiltonian does not have a bottom (but is probably bounded from above). At the points $b= \pm 1$, the bosonic metric in (3.1) vanishes and, furthermore, the matrices $\delta_{M N} \pm i \epsilon_{M N}$ entering the fermionic terms get degenerate. It is interesting to see what happens with the original superfield Lagrangian at these special points. Without loss of generality, we can choose, e.g., $b=1$. The Lagrangian (1.1) with $g_{M N}=\delta_{M N}+i \epsilon_{M N}$, $M, N=1,2$, is reduced to

$$
L_{(b=1)}=-\frac{1}{2} \int d \theta d \bar{\theta} D Z \bar{D} \bar{Z}, \quad Z:=X^{1}-i X^{2}, \quad \bar{Z}=X^{1}+i X^{2} .
$$

The (anti)chiral spinor superfields $\Psi:=D Z, \bar{\Psi}=-\bar{D} \bar{Z}, D \Psi=\bar{D} \bar{\Psi}=0$, are just the superfield strengths of the purely fermionic $\mathcal{N}=2$ supermultiplet $(\mathbf{0 , 2}, \mathbf{2})$, with $Z, \bar{Z}$ being the relevant gauge prepotentials exhibiting the gauge freedom

$$
Z \rightarrow Z+D \Omega, \quad \bar{Z} \rightarrow \bar{Z}-\bar{D} \bar{\Omega}, \quad \Omega=\omega+\theta \phi+\bar{\theta} \sigma+\theta \bar{\theta} \delta .
$$

\footnotetext{
${ }^{7}$ This $\mathcal{N}=2$ splitting of the multiplet $(\mathbf{2}, \mathbf{4}, \mathbf{2})$, is different from the splitting $(\mathbf{2}, \mathbf{4}, \mathbf{2})=(\mathbf{2}, \mathbf{2}, \mathbf{0}) \oplus(\mathbf{0}, \mathbf{2}, \mathbf{2})$, considered in [18] and yielding the standard Kählerian $\mathcal{N}=4$ supersymmetric mechanics.
} 
Here, $\omega, \delta$ and $\phi, \sigma$ are arbitrary fermionic and bosonic complex functions ${ }^{8}$. Using this gauge freedom, one can choose the "Wess-Zumino gauge"

$$
Z=\theta \lambda+\theta \bar{\theta} g, \quad \bar{Z}=-\bar{\theta} \bar{\lambda}+\theta \bar{\theta} \bar{g}
$$

in which the Lagrangian (3.16) is reduced to

$$
L_{(b=1)}=\frac{i}{2}(\bar{\lambda} \dot{\lambda}-\dot{\bar{\lambda}} \lambda)+\frac{1}{2} g \bar{g}
$$

that is the free Lagrangian of the multiplet $(\mathbf{0}, \mathbf{2}, \mathbf{2})$. Thus at the points $b= \pm 1$ we end up with the $(\mathbf{0}, \mathbf{2}, \mathbf{2})$ Lagrangian. The original two $(\mathbf{1}, \mathbf{2}, \mathbf{1})$ multiplets are combined into a complex gauge bosonic superfield which provides an alternative description of the $(\mathbf{0}, \mathbf{2}, \mathbf{2})$ multiplet. On shell $g=0$, and the relevant Hamiltonian and supercharges vanish.

\section{Hamiltonian reduction}

The geometric meaning of the model considered can be further clarified by addressing a special class of complex $n$-dimensional manifolds with the Hermitian metric

$$
h_{j \bar{k}}=g_{(j k)}+i b_{[j k]}, \quad\left(h_{j \bar{k}}\right)^{\dagger}=h_{k \bar{j}},
$$

and constraining the real functions $g_{(j k)}, b_{[j k]}$ to depend only on the real parts of the complex variables $z^{j}(j=1, \ldots, n)$.

As was mentioned above, one can define on complex manifolds $\mathcal{N}=2$ supersymmetric sigma models $[17,18]$ whose supercharges are isomorphic to the exterior holomorphic derivative and its conjugate and realize thereby Dolbeault complex. The underlying $\mathcal{N}=2$ multiplets are of the $(\mathbf{2}, \mathbf{2}, \mathbf{0})$ type, such that each complex bosonic coordinate $z^{j}$ has one complex fermionic superpartner $\psi^{j}$. In other words, Dolbeault sigma model for a manifold of complex dimension $n$ has the same number of holomorphic fermion variables as de Rham sigma model or quasicomplex sigma model on a manifold of real dimension $n$. Hilbert spaces in the former and in the latter are therefore tightly connected.

For sure, the mapping between the $(\mathbf{2}, \mathbf{2}, \mathbf{0})$ models and the Dolbeault complexes known to mathematicians and similarity between Hilbert spaces of different complexes concern quantum supercharges in the first place. However, in order to establish the correspondence between our supercharges and the Dolbeault supercharges, it is sufficient to compare only the classical versions of the two sets.

We introduce holomorphic vielbeins satisfying ${ }^{9}$

$$
\begin{aligned}
e_{k}^{a} e_{\bar{i}}^{\bar{a}}=h_{k \bar{i}}, & e_{a}^{k} e_{\bar{a}}^{\bar{i}}=h^{\bar{i} k}, & h^{\bar{i} k} h_{k \bar{j}}=\delta_{\bar{j}}^{\bar{i}}, & h_{k \bar{j}} h^{\bar{j} l}=\delta_{k}^{l}, \\
e_{k}^{a} e_{a}^{j}=\delta_{k}^{j}, & e_{a}^{k} e_{k}^{b}=\delta_{a}^{b}, & e_{\bar{k}}^{\bar{a}} e_{\bar{a}}^{\bar{j}}=\delta_{\bar{k}}^{\bar{j}}, & e_{\bar{a}}^{\bar{k}} e_{\bar{k}}^{\bar{b}}=\delta_{\bar{a}}^{\bar{b}} .
\end{aligned}
$$

The Dolbeault $\mathcal{N}=2$ supercharges were written in [18] in the form analogous to $(2.10)^{10}$,

$$
Q=\psi^{k}\left(P_{k}-i \bar{\psi}^{\bar{a}} \psi^{b} \Omega_{k, \bar{a} b}\right), \quad \bar{Q}=\bar{\psi}^{\bar{k}}\left(P_{\bar{k}}+i \bar{\psi}^{\bar{a}} \psi^{b} \bar{\Omega}_{\bar{k}, b \bar{a}}\right),
$$

where $\Omega_{j, \bar{a} b}=e_{p}^{a}\left(\partial_{j} e_{b}^{p}+\Gamma_{j k}^{p} e_{b}^{k}\right)$ are complex spin connections and the momentum $P_{M}$ is the canonical momentum calculated at fixed $\psi^{a}, \bar{\psi}^{\bar{a}}$. For further convenience, we omitted the

\footnotetext{
${ }^{8}$ The superfield gauge parameter $\Omega$ itself is defined up to addition of an arbitrary antichiral superfield, so only the gauge parameters $\phi$ and $\delta$ actually matter.

${ }^{9}$ We follow the notation of Section 2 of [18], where the reader is redirected for further details.

${ }^{10}$ See equation (3.15) of [18], where one has to set $W=0$.
} 
factors $\sqrt{2}$ compared to the expressions in [18], so the relevant Hamiltonians coincide with each other up to the factor $1 / 2$.

To establish the sought correspondence with the quasicomplex model of the preceding sections, it is convenient to rewrite the supercharges (4.1) in terms of the fermionic variables with the world indices, like in (2.8), (2.9). We obtain

$$
Q=\psi^{j}\left(\Pi_{j}+\frac{i}{2} \Gamma_{j, k \bar{p}} \psi^{k} \bar{\psi}^{\bar{p}}\right), \quad \bar{Q}=\bar{\psi}^{j}\left(\Pi_{\bar{j}}-\frac{i}{2} \Gamma_{\bar{j}, k \bar{p}} \psi^{k} \bar{\psi}^{\bar{p}}\right) .
$$

Here,

$$
\Gamma_{j, k \bar{p}}=\frac{1}{2}\left(\partial_{k} h_{j \bar{p}}-\partial_{j} h_{k \bar{p}}\right), \quad \Gamma_{\bar{j}, k \bar{p}}=\frac{1}{2}\left(\partial_{\bar{p}} h_{k \bar{j}}-\partial_{\bar{j}} h_{k \bar{p}}\right),
$$

and the momenta $\Pi_{j}, \Pi_{\bar{j}}$ are related to $P_{j}, P_{\bar{j}}$ in the same way as in (2.12),

$$
P_{j}=\Pi_{j}+\frac{i}{2}\left[\left(\partial_{j} e_{p}^{a}\right) \bar{e}_{\bar{q}}^{\bar{a}}-\left(\partial_{j} e_{\bar{q}}^{\bar{a}}\right) e_{p}^{a}\right] \psi^{p} \bar{\psi}^{\bar{q}}, \quad P_{\bar{j}}=\Pi_{\bar{j}}+\frac{i}{2}\left[\left(\partial_{\bar{j}} e_{p}^{a}\right) \bar{e}_{\bar{q}}^{\bar{a}}-\left(\partial_{\bar{j}} e_{\bar{q}}^{\bar{a}}\right) e_{p}^{a}\right] \psi^{p} \bar{\psi}^{\bar{q}} .
$$

Note that the Christoffel symbols (4.3) vanish for Kähler manifolds, killing the three-fermion terms in (4.2). This nice property is specific for the form (4.2) of the supercharges.

Requiring the metric and vielbeins to depend only on the real parts of $z^{j}$ amounts to assuming that the manifold involves isometries realized as shifts in the imaginary directions. One can observe that, in this case, the Poisson brackets of the supercharges (and hence of the Hamiltonian) with the generator of these imaginary shifts $\Pi_{j}-\Pi_{\bar{j}}=P_{j}-P_{j}$ all vanish. This allows one to perform a Hamiltonian reduction - to identify $\Pi_{j} \equiv \Pi_{\bar{j}}$ and to forget about the imaginary parts of the coordinates whatsoever.

Physically, the picture becomes more transparent at the quantum level - one can observe that the metric isometries allow one to define the Hamiltonian acting on the restricted Hilbert space, with the wave functions depending only on $\operatorname{Re} z^{j} \cdot{ }^{11}$ But, for establishing the correspondence, one can stay at the classical level.

One has a pleasure to observe that, after this identification, the expressions (4.2) coincide with (2.8). In particular, the 2-dimensional model of the previous section can be obtained by the Hamiltonian reduction from the complex Dolbeault model living on a manifold of complex dimension 2 with the metric $h_{j \bar{k}}=\delta_{j k}+i b \epsilon_{j k}$ and the vielbeins (3.3).

For completeness, we will finally discuss the same reduction directly in terms of Hamiltonians. The classical Hamiltonian of the complex $\mathcal{N}=2$ model (with the vanishing background gauge potential, i.e. with $W=0$ ) was written in [18] as

$$
H_{\mathrm{cl}}=h^{\bar{k} j}\left(P_{j}+i \hat{\Omega}_{j, \bar{b} a} \psi^{a} \bar{\psi}^{\bar{b}}\right)\left(P_{\bar{k}}-i \hat{\bar{\Omega}}_{\bar{k}, c \bar{d}} \psi^{c} \bar{\psi}^{\bar{d}}\right)-e_{a}^{t} e_{c}^{j} e_{\bar{b}}^{\bar{l}} e_{\bar{d}}^{\bar{k}}\left(\partial_{t} \partial_{\bar{l}} h_{j \bar{k}}\right) \psi^{a} \psi^{c} \bar{\psi}^{\bar{b}} \bar{\psi}^{\bar{d}},
$$

where

$$
\hat{\Omega}_{j, \bar{b} a}=-\hat{\Omega}_{j, a \bar{b}}=e_{k}^{b} \partial_{j} e_{a}^{k}+e_{\bar{b}}^{\bar{t}} e_{a}^{k} \hat{\Gamma}_{\bar{t}, j k}, \quad \hat{\Gamma}_{\bar{t}, j k}=\partial_{k} h_{j \bar{t}} .
$$

After passing everywhere to the world indices (in particular, by making use of the relation (4.4)) and identifying $\partial_{j}=\partial_{\bar{j}}$, this Hamiltonian can be rewritten as

$$
\begin{aligned}
H_{\mathrm{cl}}= & h^{\bar{k} j}\left[\Pi_{j}+i\left(\partial_{p} h_{j \bar{q}}-\frac{1}{2} \partial_{j} h_{p \bar{q}}\right) \psi^{p} \bar{\psi}^{q}\right]\left[\Pi_{k}-i\left(\partial_{p} h_{q \bar{k}}-\frac{1}{2} \partial_{k} h_{q \bar{p}}\right) \psi^{q} \bar{\psi}^{p}\right] \\
& -\partial_{t} \partial_{l} h_{j \bar{k}} \psi^{t} \psi^{j} \bar{\psi}^{l} \bar{\psi}^{k} .
\end{aligned}
$$

For the 2-dimensional target metric $h_{j \bar{k}}=\delta_{j k}+i b \epsilon_{j k}$ the last term in (4.5) vanishes and $H_{\mathrm{cl}}$ nicely coincides with (3.7), up to the overall factor $1 / 2$.

\footnotetext{
${ }^{11}$ In fact, the conditions $\Pi_{j}-\Pi_{\bar{j}}=0$ represent first class constraints, like the Gauss law constraint in standard gauge theories. Our reduced system (2.6) can thus be interpreted as a certain gauge model with the constraints resolved. At the level of off-shell superfield actions, the same reduction could presumably be accomplished using the gauging techniques of [5].
} 


\section{$5 \quad$ Summary and outlook}

In this paper, we introduced and studied a new class of $\mathcal{N}=2$ supersymmetric quantum mechanical systems, the quasicomplex quantum mechanics. Its superfield Lagrangian involves, besides the standard metric term, also an antisymmetric tensor which cannot be identified with any torsion potential. These two terms are naturally joined into a non-symmetric Hermitian target space superfield metric. In components, the antisymmetric tensor generates some nontrivial target bosonic metric even in the case when the standard metric is flat.

From the geometrical point of view, these models realize a new complex which coincides neither with the de Rham complex nor with the Dolbeault one and seems not to be discussed earlier by mathematicians. However, they are still related in a certain way to both these complexes.

- First, as we have seen, a quasicomplex sigma model living on a real $n$-dimensional manifold can be reproduced through Hamiltonian reduction from the Dolbeault $\mathcal{N}=2$ models living on a manifold of complex dimension $n$ and possessing appropriate isometries. The latter allow one to get rid of a half of real bosonic coordinates.

- Second, they may be related to a certain de Rham complex through a similarity transformation of the holomorphic quantum supercharges.

We explicitly constructed here such a transformation in the simplest two-dimensional example. However, as was recently shown [22], both the generic multidimensional quasicomplex system (2.8) and the standard de Rham system (2.9) can be related to a free system by the proper similarity transformations of the supercharges. Thus, a combination of these transformations gives a generic similarity transformation quasicomplex $\rightarrow$ de Rham, a multidimensional generalization of the transformation (3.13). Perhaps it is worth mentioning once more that this similarity of the two systems does not imply their equivalency: their Hamiltonians do not coincide and have different spectra.

We studied in detail the simplest 2-dimensional version of these unusual models and found that, at the quantum level, the spectrum involves a 4-fold degeneracy of the states, thus exhibiting a hidden $\mathcal{N}=4$ supersymmetry. Studying (in Appendix B) the spectrum further, we discovered its rather interesting features. In a certain limit, the spectrum consists of $\mathfrak{s u}(2)$ multiplets with half-integer momenta in both fermionic and bosonic sectors. The Hamiltonian is related by a similarity transformation to the square of the Dirac operator, $H=\not^{2} .^{12}$

Studying the spectra of more complicated quasicomplex Hamiltonians in higher dimensions would be highly desirable.

\section{A Relation to $\mathcal{N}=1, d=2$ sigma model}

Here we will consider the one-dimensional reduction of the most general superfield Lagrangian of the $\mathcal{N}=1, d=2$ sigma model associated with the real superfield $X^{M}$.

Following [13], such a Lagrangian in the light-cone parametrization of the $\mathcal{N}=1, d=2$ superspace can be written as

$$
\begin{aligned}
L_{(d=2)}= & \int d \theta^{+} d \theta^{-}\left\{G_{(M N)} D_{+} X^{M} D_{-} X^{N}+B_{[M N]} D_{+} X^{M} D_{-} X^{N}\right. \\
& \left.+G_{[M N]}^{--} D_{-} X^{M} D_{-} X^{N}+G_{[M N]}^{++} D_{+} X^{M} D_{+} X^{N}\right\},
\end{aligned}
$$

where $\theta^{ \pm}$are real Grassmann coordinates, $D_{ \pm}=\frac{\partial}{\partial \theta^{ \pm}}+i \theta^{ \pm} \partial_{ \pm \pm},\left(D_{ \pm}\right)^{2}=i \partial_{ \pm \pm}, \overline{\left(D_{ \pm}\right)}=-D_{ \pm}$, and $G_{(M N)}(X), B_{[M N]}(X), G_{[M N]}^{ \pm \pm}(X)$ are real functions. Since $X^{M}$ are $d=2$ Lorentz scalars,

\footnotetext{
${ }^{12}$ We want to stress that in this case we are talking about the similarity transformation of the Hamiltonians that gives the equivalence of the spectra, mapping of the wave functions, etc.
} 
the last two terms in (A.1) explicitly break $d=2$ Lorentz covariance. The Lagrangian (A.1) exhibits invariance under the target space diffeomorphisms realized like in (2.5) and gauge transformations associated with the antisymmetric field $B_{[M N]}$

$$
\delta B_{[M N]}(X)=\partial_{M} A_{N}(X)-\partial_{N} A_{M}(X),
$$

where $A_{N}(X)$ are arbitrary real parameters. The $d=2$ Lorentz breaking tensors $G_{[M N]}^{ \pm \pm}$do not introduce any additional target space gauge freedom.

Now we pass to the complex coordinates $\theta=\theta^{+}+i \theta^{-}, \bar{\theta}=\theta^{+}-i \theta^{-}$, and the complex spinor derivatives

$$
\begin{aligned}
& D_{+}=D-\bar{D}, \quad D_{-}=i(D+\bar{D}), \\
& \{D, D\}=\{\bar{D}, \bar{D}\}=\frac{i}{2}\left(\partial_{++}-\partial_{--}\right), \quad\{D, \bar{D}\}=-\frac{i}{2}\left(\partial_{++}+\partial_{--}\right) .
\end{aligned}
$$

In terms of $D, \bar{D}$ the Lagrangian (A.1), up to an overall numerical coefficient, can be rewritten as

$$
\begin{aligned}
\int d \bar{\theta} & d \theta\left\{\left(g_{(M N)}+i b_{[M N]}\right) D X^{M} \bar{D} X^{N}\right. \\
+ & \left.\frac{1}{2} \mathcal{B}_{[M N]} D X^{M} D X^{N}-\frac{1}{2} \overline{\mathcal{B}}_{[M N]} \bar{D} X^{M} \bar{D} X^{N}\right\}
\end{aligned}
$$

where

$$
\begin{aligned}
& g_{(M N)}=G_{(M N)}, \quad b_{[M N]}=G_{[M N]}^{++}+G_{[M N]}^{--}, \\
& \mathcal{B}_{[M N]}=B_{[M N]}-i\left(G_{[M N]}^{++}-\left(G_{[M N]}^{--}\right), \quad \overline{\mathcal{B}}_{[M N]}=B_{[M N]}+i\left(G_{[M N]}^{++}-\left(G_{[M N]}^{--}\right) .\right.\right.
\end{aligned}
$$

After performing the $d=2 \rightarrow d=1$ dimensional reduction as $\partial_{++}=\partial_{--}=-2 \partial_{t}$, the spinor derivatives (A.3) become the $\mathcal{N}=2, d=1$ spinor derivatives and (A.4) is recognized as the Lagrangian (1.1) with the non-symmetric Hermitian metric (2.1) modified by terms with extra antisymmetric tensor fields $\mathcal{B}_{[M N]}, \overline{\mathcal{B}}_{[M N]}$. These objects are gauge potentials of the torsion on the target space [10], with the same gauge transformation law as in (A.2). The field $b_{[M N]}$ does not bring in any new target space gauge freedom like its $d=2$ prototypes $G_{[M N]}^{ \pm \pm}$.

\section{B Hamiltonian (3.12) on $S^{2}$ and its spectrum}

Consider first the Hamiltonian in the sector $F=0$ written in equation (3.15). Introduce the complex coordinates,

$$
\partial_{1}=\frac{\mu}{\sqrt{2}}\left(\partial_{w}+\partial_{\bar{w}}\right), \quad \partial_{2}=\frac{i \mu}{\sqrt{2}}\left(\partial_{\bar{w}}-\partial_{w}\right) .
$$

The $S^{2}$ case is obtained by identifying

$$
1-b^{2}=\frac{\rho}{(1+w \bar{w})^{2}}
$$

( $\mu$ and $\rho$ are arbitrary real constants). This implies

$$
b= \pm \frac{\sqrt{(1+X)^{2}-\rho}}{1+X}, \quad b^{\prime}= \pm \frac{\rho}{(1+X)^{2} \sqrt{(1+X)^{2}-\rho}}, \quad X:=w \bar{w} .
$$


Choosing in (B.1) the positive sign and substituting it into (3.15), we obtain the Hamiltonian

$$
H_{S^{2}}=\frac{\mu^{2}}{\rho}\left[-(1+w \bar{w})^{2} \partial \bar{\partial}-\frac{1}{2}(1+w \bar{w})(w \partial+\bar{w} \bar{\partial})+\frac{1}{2} \frac{(1+w \bar{w})^{2}}{\sqrt{(1+w \bar{w})^{2}-\rho}} J\right],
$$

where

$$
J=w \partial-\bar{w} \bar{\partial}
$$

is the charge operator which commutes with the whole $H_{S^{2}}$.

The Hamiltonian (B.2) is Hermitian with the measure $\sqrt{1-b^{2}} \propto 1 /(1+w \bar{w})$ (see the remark after (3.14)). Thus, the Hilbert space where this Hamiltonian acts involves the wave functions normalized as

$$
\int|\Psi(w, \bar{w})|^{2} \frac{d w d \bar{w}}{1+w \bar{w}}=1 .
$$

It is $1+w \bar{w}$ downstairs, not $(1+w \bar{w})^{2}$ as for the standard Laplacian on $S^{2}$ !

In the sector $J=0$, the Hamiltonian acquires a particularly simple form, such that the spectrum and wave functions can be found analytically. Indeed, introducing the variable $z=$ $\frac{1-X}{1+X}$ (it is none other than the cosine of the polar angle on $S^{2}$ ), the Schrödinger equation acquires in this sector the form

$$
\left(z^{2}-1\right) \Psi^{\prime \prime}(z)+(z+1) \Psi^{\prime}(z)=E \Psi(z) .
$$

The solutions are Jacobi polynomials,

$$
\Psi_{m}(z)=(1+z) P_{m}^{0,1}(z), \quad m=1, \ldots,
$$

which gives the spectrum

$$
E_{m}=m^{2} .
$$

Note that we have excluded the function $\Psi=$ const (which is a formal solution of (B.5)), because it is not normalizable with the measure in (B.4). This means that the zero-energy states are absent in the spectrum and supersymmetry is thus broken. The first few states are

$$
\begin{aligned}
& \Psi_{1}(z)=1+z, \quad \Psi_{2}(z)=(1+z)(1-3 z), \\
& \Psi_{3}(z)=(1+z)\left(1+2 z-5 z^{2}\right), \quad \ldots
\end{aligned}
$$

When $J \neq 0$, the situation is more complicated. The relevant wave functions are eigenfunctions of $J$ with integer non-zero eigenvalues. We pose

$$
\Psi_{J}=w^{J} f_{J}(z)(J>0), \quad \Psi_{J}=\bar{w}^{|J|} g_{J}(J<0) .
$$

The Schrödinger equation for $\Psi_{J>0}$ is reduced to the following equation for $f_{J}$,

$$
\left(z^{2}-1\right) f_{J}^{\prime \prime}+(1+2 J+z) f_{J}^{\prime}-\frac{J}{1+z}\left(1-\frac{1}{\sqrt{1-\rho \frac{(1+z)^{2}}{4}}}\right) f_{J}=E f_{J} .
$$

The function $g_{J}$ satisfies the similar equation with $J$ being replaced by $|J|$ and with the opposite sign before the second term in the round brackets. These equations can be solved numerically, when imposing proper boundary conditions. 
In the sector $F=2$, the Hamiltonian is the same as in (B.2) up to the opposite sign for the last term. The spectrum is the same as for $F=0$, but with the complex conjugated wave functions. The states in the sector $F=1$ are obtained by the action of the supercharges.

Notice now that the problem is drastically simplified in the limit $\rho, \mu \rightarrow 0$, the ratio $\mu^{2} / \rho$ being kept fixed. Let the latter be 1 . The Hamiltonian acquires the form

$$
H_{(\rho=0)}=-(1+w \bar{w})^{2} \partial \bar{\partial}-(1+w \bar{w}) \bar{w} \bar{\partial} .
$$

One can observe now that this Hamiltonian commutes not only with (B.3), but also with the operators

$$
J_{+}=\partial+\bar{w}^{2} \bar{\partial}, \quad J_{-}=\bar{\partial}+w^{2} \partial+w .
$$

They are mutually (anti)conjugated with respect to the measure $\sim \frac{1}{1+w \bar{w}}$ and form the $\mathfrak{s u}(2)$ algebra,

$$
\left[J_{+}, J_{-}\right]=2 J_{3}, \quad\left[J_{ \pm}, J_{3}\right]= \pm J_{ \pm}
$$

with $J_{3}=J+1 / 2$.

This means that the eigenstates of (B.9) represent $\mathrm{SU}(2)$ multiplets. An amusing fact is that, in contrast to the case of the ordinary Laplacian, $\triangle=(1+\bar{w} w)^{2} \partial \bar{\partial}$, these multiplets correspond to half-integer momenta. Indeed, in the ground state (with $E=1$ ), we find a doublet,

$$
\Psi_{0}=\frac{1}{1+w \bar{w}}, \quad \Psi_{+}=\frac{\bar{w}}{1+w \bar{w}} .
$$

These states are obtained from one another by the action of $J_{+}$and $J_{-}$. At the level $E=4$, we have four states,

$$
\Psi_{-}=\frac{w}{(1+w \bar{w})^{2}}, \quad \Psi_{0}=\frac{1-2 w \bar{w}}{(1+w \bar{w})^{2}}, \quad \Psi_{+}=\frac{\bar{w}(2-w \bar{w})}{(1+w \bar{w})^{2}}, \quad \Psi_{++}=\frac{\bar{w}^{2}}{(1+w \bar{w})^{2}} .
$$

Note that the $J=0$ reduction of the general Hamiltonian (B.2) exactly coincides with the reduction of (B.9) since $w \partial_{w}=\bar{w} \partial_{\bar{w}}$ on the $J=0$ wave functions. Hence (B.9) has the same energy spectrum given by (B.6) and all neutral components of the above $\mathrm{SU}(2)$ multiplets are simultaneously eigenfunctions of the generic $J=0$ Hamiltonian. In particular, the neutral functions $\Psi_{0}$ in (B.11) and (B.12) coincide, up to numerical coefficients, with $\Psi_{1}$ and $\Psi_{2}$ in the sequence (B.7). Posing $m=s+1 / 2$, we represent the spectrum (B.6) as

$$
E_{s}=s(s+1)+1 / 4
$$

with the multiplicity $2 s+1$. The Hamiltonian (B.9) represents the Casimir operator of the algebra $\left\{J_{ \pm}, J_{3}\right\}$ shifted by a constant. Indeed, it is easy to directly check that for the realization (B.10)

$$
C_{2}=-\frac{1}{2}\left[J_{+} J_{-}+J_{-} J_{+}-2\left(J_{3}\right)^{2}\right]=H_{(\rho=0)}-\frac{1}{4} .
$$

One can recall now that the spectrum (B.13) is also characteristic for the Dirac operator on $S^{2}$ in the case when the gauge field is absent and only spin connections are taken into account (see, e.g., [1]). And, indeed, one can observe that the Hamiltonian (B.9) is related to the square of the Dirac operator multiplied by the positive chirality projector, $H_{D}^{(+)}=\not^{2} \frac{1+\sigma_{3}}{2}$ and expressed via complex variables $w, \bar{w}$ by a similarity transformation,

$$
H_{(\rho=0)}=\frac{1}{\sqrt{1+w \bar{w}}} H_{D}^{(+)} \sqrt{1+w \bar{w}} .
$$


This transformation changes the measure: the eigenfunctions of $H_{D}$ are normalized with the standard measure $\propto 1 /(1+w \bar{w})^{2}$, while the eigenfunctions of $H_{(\rho=0)}$ are normalized as in (B.4). For instance, the wave functions (B.11) correspond to the following set of the spin $1 / 2$ eigenfunctions for $H_{D}^{(+)}$:

$$
\Psi_{0}^{(+)}=\frac{1}{\sqrt{1+w \bar{w}}}, \quad \Psi_{+}^{(+)}=\frac{\bar{w}}{\sqrt{1+w \bar{w}}} .
$$

The same similarity transformation transforms $H_{D}^{(-)}=\mathcal{D}^{2} \frac{1-\sigma_{3}}{2}$ to the Hamiltonian

$$
H_{(\rho=0)}^{\prime}=-(1+w \bar{w})^{2} \partial \bar{\partial}-(1+w \bar{w}) w \partial,
$$

which coincides with the Hamiltonian of our model in the sector $F=2$ in the limit $\rho \rightarrow 0$ ! As was discussed in Section 3, it has the same spectrum as (B.9) with the complex conjugated wave functions. The corresponding realization of the $\mathrm{SU}(2)$ generators is as follows

$$
J_{+}^{\prime}=\partial+\bar{w}^{2} \bar{\partial}+\bar{w}, \quad J_{-}^{\prime}=\bar{\partial}+w^{2} \partial, \quad J_{3}^{\prime}=J-\frac{1}{2} .
$$

For completeness, it is instructive to explicitly give how the Hamiltonians $H_{D}^{( \pm)}$look in our notation,

$$
H_{D}^{( \pm)}=-(1+w \bar{w})^{2} \partial \bar{\partial} \pm \frac{1}{2}(1+w \bar{w})\left(w \partial_{w}-\bar{w} \partial_{\bar{w}} \pm \frac{1}{2}\right)+\frac{1}{4} .
$$

The relevant realizations of the $\mathrm{SU}(2)$ generators are obtained from (B.10) and (B.14) by the same similarity transformation:

$$
J_{+}^{( \pm)}=\partial+\bar{w}^{2} \bar{\partial} \mp \frac{1}{2} \bar{w}, \quad J_{-}^{( \pm)}=\bar{\partial}+w^{2} \partial \pm \frac{1}{2} w, \quad J_{3}^{( \pm)}=J \pm \frac{1}{2} .
$$

To understand better what happens, let us look at the supercharges. In the limit $\mu, \rho \rightarrow 0$, the supercharge $Q$ in (3.11) depends only on one holomorphic fermion variable $\chi=\left(\psi_{1}+i \psi_{2}\right) / \sqrt{2}$. Similarly, $\bar{Q}$ depends only on $\bar{\chi}$ :

$$
Q=-i \sqrt{2} \chi(1+w \bar{w}) \bar{\partial}, \quad \bar{Q}=-i \sqrt{2} \bar{\chi}(1+w \bar{w}) \partial .
$$

The similarity transformation

$$
(Q, \bar{Q}) \Rightarrow \sqrt{1+w \bar{w}}(Q, \bar{Q}) \frac{1}{\sqrt{1+w \bar{w}}}=(\tilde{Q}, \overline{\tilde{Q}})
$$

(the same for $Q$ and $\bar{Q} !)$ gives the supercharges

$$
\tilde{Q}=-i \sqrt{2} \chi(1+w \bar{w})\left[\bar{\partial}-\frac{w}{2(1+w \bar{w})}\right], \quad \overline{\tilde{Q}}=-i \sqrt{2} \bar{\chi}(1+w \bar{w})\left[\partial-\frac{\bar{w}}{2(1+w \bar{w})}\right] .
$$

There is only one holomorphic fermion variable here, and the system can be described in terms of the $(\mathbf{2}, \mathbf{2}, \mathbf{0})$ superfield. The supercharges (B.16) coincide (if identifying $w \equiv \bar{z}$ ) with those in equation (3.26) of [18] brought on the sphere, with setting $W=0$ (no gauge field). After mapping $\psi_{1,2} \rightarrow \sigma_{1,2} / \sqrt{2}$, the supercharges (B.16) are mapped onto $\not{D} \frac{1 \pm \sigma_{3}}{2}$.

Coming back to the spectrum of the full supersymmetric Hamiltonian in the limit $\rho \rightarrow 0$, it represents two copies of the spectrum of the system (B.15). Indeed, the full Hilbert space involves the functions $\Psi(w, \bar{w}, \chi, \lambda)$ where $\lambda=\left(\psi_{1}-i \psi_{2}\right) / \sqrt{2}$ is the holomorphic fermion variable orthogonal to $\chi$. Thus, one can, e.g., multiply the fermion state of the system (B.15) by $\lambda$ to obtain the state in the sector $F=2$ of the full Hamiltonian with the same energy.

Note finally that such a nice interpretation reducing the problem to something already known is possible only in the limit $\rho \rightarrow 0$. When $\rho \neq 0$, the spectrum of the states with $J \neq 0$ does not coincide with (B.6) (this is best seen if assuming $\rho$ in equation (B.8) to be small and finding out that the perturbative corrections $\propto \rho$ to the spectrum (B.6) do not vanish) and we obtain something new. 


\section{Acknowledgements}

We are indebted to S. Fedoruk for useful discussions. E.I. would like to thank SUBATECH, Université de Nantes, for the warm hospitality in the course of this study. His work was carried out under the Convention $\mathrm{N}^{\circ} 2010$ 11780. He also acknowledges support from the RFBR grants 09-01-93107, 11-02-90445, 12-02-00517 and a grant of the IN2P3-JINR Programme for 2012.

\section{References}

[1] Abrikosov Jr. A.A., Dirac operator on the Riemann sphere, hep-th/0212134.

[2] Chamseddine A.H., Complexified gravity in noncommutative spaces, Comm. Math. Phys. 218 (2001), 283292, hep-th/0005222.

[3] Chamseddine A.H., Mukhanov V., Gravity with de Sitter and unitary tangent groups, J. High Energy Phys. 2010 (2010), no. 3, 033, 18 pages, arXiv:1002.0541.

[4] Coles R.A., Papadopoulos G., The geometry of the one-dimensional supersymmetric nonlinear sigma models, Classical Quantum Gravity 7 (1990), 427-438.

[5] Delduc F., Ivanov E., Gauging $\mathcal{N}=4$ supersymmetric mechanics, Nuclear Phys. B 753 (2006), 211-241, hep-th/0605211.

[6] Dunne G.V., Jackiw R., Trugenberger C.A., "Topological" (Chern-Simons) quantum mechanics, Phys. Rev. D 41 (1990), 661-666.

[7] Einstein A., A generalization of the relativistic theory of gravitation, Ann. of Math. 46 (1945), 578-584.

[8] Einstein A., Straus E.G., A generalization of the relativistic theory of gravitation. II, Ann. of Math. 47 (1946), 731-741.

[9] Faddeev L., Jackiw R., Hamiltonian reduction of unconstrained and constrained systems, Phys. Rev. Lett. 60 (1988), 1692-1694.

[10] Fedoruk S.A., Ivanov E.A., Smilga A.V., Real and complex supersymmetric $d=1$ sigma models with torsions, Internat. J. Modern Phys. A 27 (2012), 1250146, 25 pages, arXiv:1204.4105.

[11] Floreanini R., Percacci R., Sezgin E., Sigma models with purely Wess-Zumino-Witten actions, Nuclear Phys. B 322 (1989), 255-276.

[12] Freedman D.Z., Townsend P.K., Antisymmetric tensor gauge theories and nonlinear $\sigma$-models, Nuclear Phys. B 177 (1981), 282-296.

[13] Gibbons G.W., Papadopoulos G., Stelle K.S., HKT and OKT geometries on soliton black hole moduli spaces, Nuclear Phys. B 508 (1997), 623-658, hep-th/9706207.

[14] Giveon A., Porrati M., Rabinovici E., Target space duality in string theory, Phys. Rep. 244 (1994), 77-202, hep-th/9401139.

[15] Hohm O., Hull C., Zwiebach B., Generalized metric formulation of double field theory, J. High Energy Phys. 2010 (2010), no. 8, 008, 35 pages, arXiv:1006.4823.

[16] Howe P.S., Townsend P.K., Chern-Simons quantum mechanics, Classical Quantum Gravity 7 (1990), 16551668.

[17] Hull C.M., The geometry of supersymmetric quantum mechanics, hep-th/9910028.

[18] Ivanov E.A., Smilga A.V., Dirac operator on complex manifolds and supersymmetric quantum mechanics, Internat. J. Modern Phys. A 27 (2012), 1230024, 30 pages, arXiv:1012.2069.

[19] Pashnev A., Toppan F., On the classification of $N$-extended supersymmetric quantum mechanical systems, J. Math. Phys. 42 (2001), 5257-5271, hep-th/0010135.

[20] Smilga A.V., How to quantize supersymmetric theories, Nuclear Phys. B 292 (1987), 363-380.

[21] Smilga A.V., SUSY anomaly in quantum-mechanical systems, Phys. Lett. B 199 (1987), 516-518.

[22] Smilga A.V., Taming the zoo of supersymmetric quantum mechanical models, J. High Energy Phys. 2013 (2013), no. 5, 119, 23 pages, arXiv:1301.7438.

[23] Witten E., Constraints on supersymmetry breaking, Nuclear Phys. B 202 (1982), 253-316.

[24] Witten E., Supersymmetry and Morse theory, J. Differential Geom. 17 (1982), 661-692. 